\title{
Primary immune surveillance: some like it hot
}

\author{
Joseph J. Skitzki • Qing Chen - W. C. Wang • \\ Sharon S. Evans
}

Received: 17 April 2007 /Revised: 11 June 2007 / Accepted: 29 June 2007 / Published online: 18 August 2007

(C) Springer-Verlag 2007

\begin{abstract}
The thermal element of fever has been found to be beneficial in models of infectious disease. The contributions of fever-range temperatures to the efficacy of the adaptive immune response have only begun to be delineated. There is accumulating evidence that fever-range thermal stress bolsters primary immune surveillance of lymph nodes and Peyer patches by augmenting lymphocyte extravasation across specialized vessels termed high endothelial venules. Molecular mechanisms have recently come to light by which the thermal component of fever alone may promote lymphocyte trafficking, and thereby the probability of mounting a defense against microbial infection. Acquired knowledge of the molecular changes associated with thermal stress may allow for the development of novel therapies for a variety of disease processes.
\end{abstract}

Keywords Hyperthermia - Lymphocyte trafficking ·

Primary immune surveillance $\cdot$ IL-6

\section{Introduction}

Fever is one of the most common but least understood of the biological processes associated with inflammation [1]. Debate continues over both the potential harms and benefits afforded by this physiologic function. In terms of evolution, fever is highly conserved despite the associated increase in metabolic demands. Evidence for the benefits of fever is noted in both homeothermic (warm-blooded) and

J. J. Skitzki • Q. Chen · W. C. Wang · S. S. Evans $(\bowtie)$

Department of Immunology, Roswell Park Cancer Institute,

Elm \& Carlton Streets,

Buffalo, NY 14263, USA

e-mail: sharon.evans@roswellpark.org

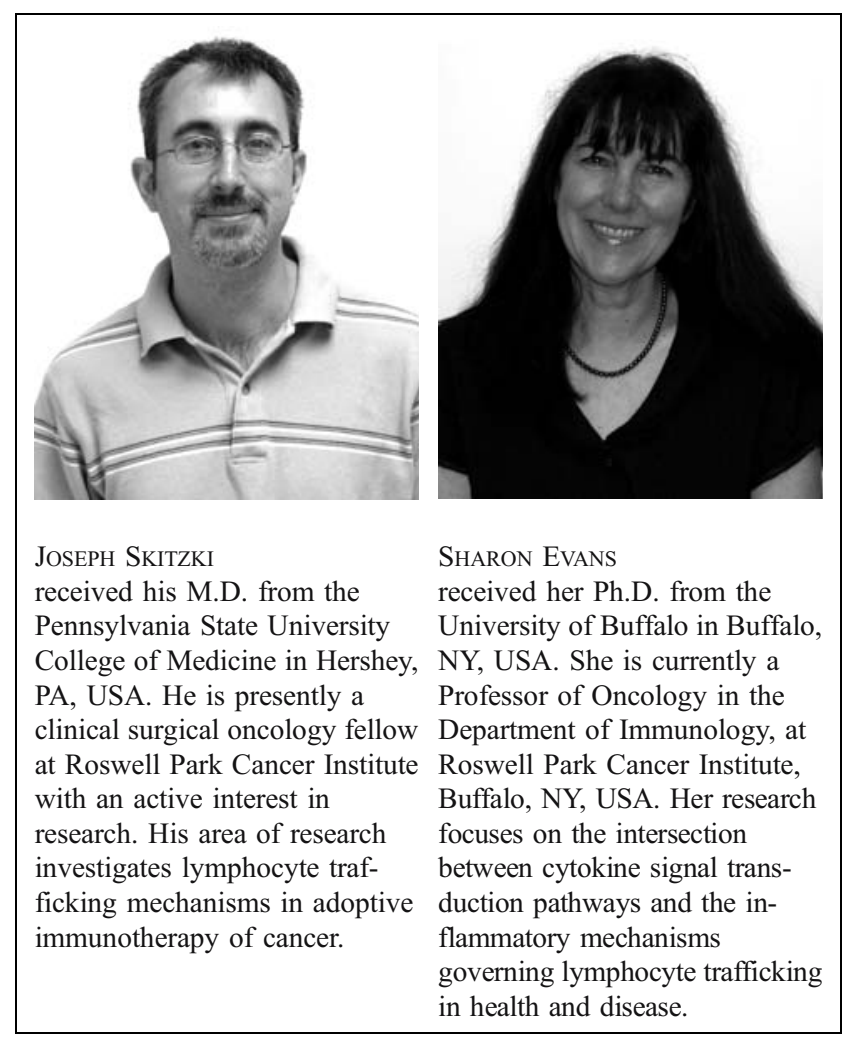

poikilothermic (cold-blooded) animals. Experiments have demonstrated the ability of poikilotherms to alter their behavior during infection and migrate to warmer environments, thereby elevating their core body temperatures $[2$, 3]. This heat-seeking behavior has been linked to improved survival $[2,4,5]$ and could be abrogated with the use of antipyretics [6]. Homeothermic mammals also demonstrate improved survival with fever in models of infection. Multiple studies in mice, rabbits, and dogs have shown similar findings of increased survival even when body 
temperatures are artificially increased to the range of natural fever $[3,7,8]$. However, fever may be detrimental to the severely ill who cannot afford the increased metabolic demands $[7,9,10]$. Given this paradigm, fever may be viewed as a beneficial adaptive response that has persisted over millions of years. The role of the thermal component of fever in regulating the immune system has only begun to be elucidated. The following review will detail the potential contributions of the thermal element of fever to primary immune surveillance.

\section{Fever and hyperthermia in the context of disease}

Before discussing temperature and its effect on the immune system, it is necessary to distinguish between the terms fever and hyperthermia. Fever is defined as a state of elevated core temperature as a defensive response of a host to pathogenic stimuli. In fever, elevation of the set point of body temperature increases core temperature via thermoeffectors such as prostaglandins or cytokines [11]. This response is accompanied by a complex interplay of cytokine networks and hemodynamic parameters such as vasodilatation and blood flow. Elevations in body temperature not associated with a change in the set point or thermoeffectors is called hyperthermia. Hyperthermia can be induced by increasing the heat load and/or inactivating heat dissipation by a variety of methods [11]. Fever is a complex state that presents difficulty in dissecting the biological changes associated with its thermal component. Given this complexity, a reductionist approach has been taken in a number of studies to investigate the biological effects of the thermal component of fever [7, 12]. Thus, experimentally induced hyperthermia differs from fever generation in organisms and avoids any confounding variables associated with natural fever such as endotoxemia, inflammatory cytokine elaboration, and/or neurohormonal axis changes. Accordingly, induced hyperthermia can be a useful tool to dissect the role of heat as one aspect of fever and its influence upon the immune system, specifically immune surveillance.

Immune surveillance by $\mathrm{T}$ and $\mathrm{B}$ lymphocytes of the adaptive immune system allows for a range of effector responses as well as the generation of memory to pathogenic stimuli. Primary immune surveillance refers to the ability of antigen-presenting cells and naïve lymphocytes to colocalize in the unique environment of peripheral lymph nodes (PLNs), which function as the first line of defense against microbial invaders entering the skin [13]. For the purposes of this review, the gut interface consisting of Peyer patches (PPs) and mesenteric lymph nodes (MLNs) as sentinels of pathogens will also be considered as components of primary immune surveillance. Dendritic cells that function as professional antigen-presenting cells enter lymph nodes via afferent lymphatics. Concurrently, naïve $\mathrm{T}$ cells continually enter through high endothelial venules (HEVs) of lymph nodes in an attempt to enhance the incredibly small chance that a particular cognate antigen will be encountered by numerically rare $T$ cells that express an appropriate $\mathrm{T}$ cell receptor [14]. Upon presentation of an appropriate antigen, antigen-specific naïve $\mathrm{T}$ cells can undergo activation, expansion, and differentiation into memory or effector phenotypes [13]. Given the grossly abundant number of potential pathogens in the external environment, the extensive recirculation of a diverse but finite number of naïve lymphocytes into the lymph nodes has evolved into a highly efficient and necessary component of the adaptive immune response. Notably, the mechanisms of lymphocyte entry into lymph nodes via HEVs are pivotal for the efficiency of this system in both normothermic and hyperthermic states.

\section{Process of lymphocyte entry into lymph nodes}

Lymphocyte entry into lymph nodes is a highly regulated process orchestrated by adhesion molecules on both lymphocytes and vascular endothelium. The high shear force exerted on lymphocytes traveling through the bloodstream makes their arrest and eventual extravasation into tissues a truly remarkable process. The multistep scheme of lymphocyte trafficking involves four consecutive interdependent steps consisting of tethering/rolling, chemokine activation, firm adhesion, and transendothelial migration $[14,15]$. The ability of lymphocytes to roll along the endothelium and slow their velocity is mediated by the presence of selectinhoming receptors. In the case of blood-borne lymphocytes entering PLNs via HEVs, lymphocyte expression of Lselectin is required for initial tethering and rolling. L-selectin present on the cell surface of lymphocytes interacts with the glycoprotein complex known as PLN addressin (PNAd) displayed on the lumenal border of HEVs [16]. In the case of MLNs or PPs of the gut, $\alpha 4 \beta 7$ integrin expressed on lymphocytes interacts with mucosal addressin cell adhesion molecule-1 (MAdCAM-1) to generate rolling.

Transient rolling interactions allow the lymphocyte to "sample" the endothelium for factors that can promote firm arrest. Activation of lymphocytes to induce firm arrest is mediated by tissue-specific chemokines displayed on endothelial surfaces [17]. In mice, HEVs have the ability to produce and display CCL21, the chemokine primarily associated with naïve lymphocyte activation $[18,19]$. In a truly restricted fashion, CCL21 has the ability to only interact with its specific receptor namely, CCR7, which is uniformly found on naïve and central memory $\mathrm{T}$ cells. Engagement of CCR7 leads to a rapid change in the affinity 
and/or avidity of lymphocyte integrins for endothelial receptors that mediate firm adhesion. The $\beta 2$-integrin, lymphocyte function-associated antigen-1 (LFA-1), on lymphocytes binds strongly with the intercellular adhesion molecule 1 or 2 (ICAM-1, ICAM-2) endothelial receptors expressed on HEVs. Upon integrin binding and firm arrest of the lymphocyte, extravasation into the lymphoid tissue occurs, which is also thought to involve ICAM-1 [20].

The elegant cascade of aforementioned events that lead to colocalization of lymphocytes and antigen-presenting cells in the compartmentalized environment of the lymph node is crucial for a successful adaptive immune response. Once in the lymph node, $\mathrm{T}$ and $\mathrm{B}$ cells migrate to their respective zones and interactions between the two populations are believed to be chemokine mediated. $\mathrm{T}$ cell activation by antigen-presenting cells that enter via afferent lymphatics subsequently occurs with the generation of a potent $\mathrm{T}$ and $\mathrm{B}$ cell response.

\section{Enhanced lymphocyte-endothelial interactions with hyperthermia}

As a nontoxic treatment, whole-body hyperthermia (WBH) maintains an elevation of core body temperature by isolating an organism in a contained, temperaturecontrolled environment, thereby mimicking the thermal component of fever $[12,21,22]$. As previously stated, elevated core body temperatures in the febrile range have been associated with improved survival in homeotherms and poikilotherms; however, alterations in the immune system during thermal stress were largely undefined. Feverrange thermal stress has previously been shown to increase lymphocyte priming, proliferation, and cytotoxicity $[2,21$, 23]. WBH also shortens the time to generate an immune response by stimulating the migration of skin-derived dendritic cells (i.e., Langerhans cells) to lymph nodes where they colocalize with arriving $T$ lymphocytes [21, 24]. Recently, lymphocyte recruitment to lymph nodes and PPs, a key component of primary immune surveillance, has been demonstrated to improve with the application of feverrange WBH $[25,26]$.

Augmentation of primary immune surveillance by feverrange thermal stress is mediated by changes found both in the lymphocyte as well as the endothelium (Fig. 1). In vivo experiments in both mice and humans demonstrated a redistribution of lymphocytes after exposure to $6 \mathrm{~h}$ of WBH in the febrile range $\left(39.5 \pm 0.5^{\circ} \mathrm{C}\right)$ with a transient decrease in the peripheral blood pool $[26,27]$. Further utilizing the murine model, lymphocytes expressing either L-selectin or $\alpha 4 \beta 7$ integrin decreased in the peripheral blood and concomitantly increased in PLNs and PPs. To determine if this redistribution was causally related to improved inter-

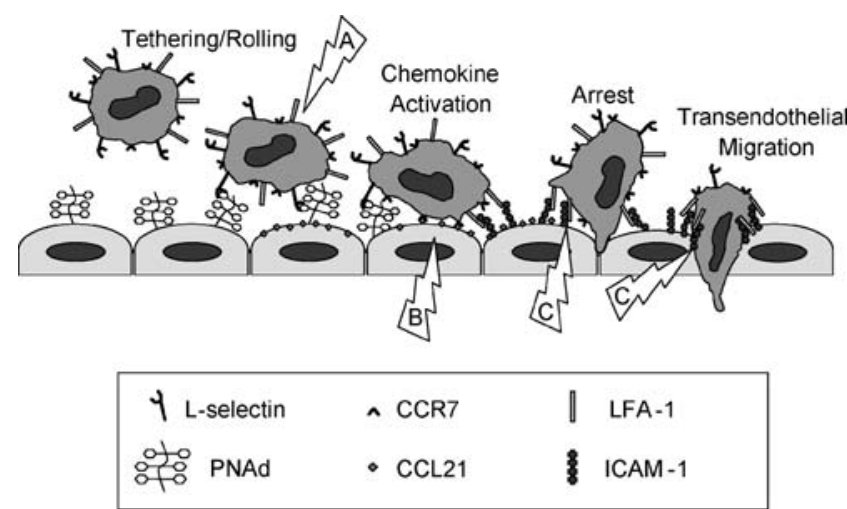

Fig. 1 Effects of fever-range hyperthermia on lymphocyte-endothelial adhesion. A, Hyperthermia increases the affinity of L-selectin expressed on lymphocytes, allowing for greater interaction with PNAd and improved tethering/rolling. $B$, Hyperthermia increases the display of CCL21 on the HEV lumenal surface to augment activation of lymphocytes via CCR7 for eventual arrest. $C$, ICAM-1 expression on HEVs is upregulated by hyperthermia to promote firm adhesion and subsequent extravasation of LFA-1-expressing lymphocytes

actions with HEVs, splenocytes taken from mice receiving WBH were examined in adhesion assays utilizing cryosections of lymphoid tissue containing HEVs, i.e., StamperWoodruff assay [28]. A significant increase was noted in the ability of splenocytes derived from WBH-treated animals to adhere to HEVs in frozen sections compared to splenocytes from normothermic control mice.

The improved adhesion noted in PLN and PP HEVs was due to L-selectin/PNAd and $\alpha 4 \beta 7 /$ MAdCAM-1-mediated primary tethering and rolling interactions, respectively, which could be abrogated with the use of adhesionblocking antibodies [26]. It is interesting to note that the improved interactions were not due to a change in the surface density of either L-selectin or $\alpha 4 \beta 7$ integrin, but rather an enhanced affinity and/or avidity, suggestive of structural changes of these molecules [29-31]. These in vitro findings were further supported by in vivo lymphocyte homing studies. Hyperthermia treatment of L-selectin or $\alpha 4 \beta 7$ integrin-expressing lymphocytes in culture, before adoptive transfer into mice, yielded an approximate twofold increase in adoptively transferred cells found in PLNs or PPs, respectively [26, 29]. Notably, thermal treatment did not override the intrinsic specificity of homing molecules as evidenced by the restricted entry of $\alpha 4 \beta 7$ integrin $^{+} / \mathrm{L}-$ selectin ${ }^{-}$cells only in PPs and not PLNs [26].

Fever-range thermal stress has also been shown to act independently upon HEVs to increase their adhesive properties as observed in multiple murine strains $[25,26]$. In vitro Stamper-Woodruff assays using PLNs or PPs of mice treated with WBH $\left(39.5 \pm 0.5^{\circ} \mathrm{C}\right.$ for $\left.6 \mathrm{~h}\right)$ demonstrated improved ability of HEVs to support lymphocyte adhesion compared to normothermic controls. Importantly, increased adhesion was only noted in the plump, near-cuboidal, 
differentiated HEVs of LNs and PPs but not in the squamous endothelium of nonlymphoid tissues [26]. In vivo homing studies also demonstrated a twofold increase in trafficking of normothermic lymphocytes to PLNs, PPs, and MLNs in mice receiving WBH. Paralleling the results of frozen section adherence assays, heat effects on homing were site specific, supported by the lack of an increase in homing to sites with normal squamous endothelial vasculature such as the liver or pancreas [25, 26, 32].

To further pinpoint the specific adhesive mechanisms that occur in cells lining HEVs, intravital microscopy studies were performed. Interactions between fluorescenttagged lymphocytes and vascular substrates in PLNs of living mice demonstrated a functional dichotomy between the effects of thermal stress on higher-order HEVs and lower-order venules [25]. Although the rolling interactions did not manifest any change, the number of lymphocytes achieving firm arrest increased with $\mathrm{WBH}$ and was restricted to the higher-order HEVs [25]. Lymphocyte aggregates were not detected in vivo, consistent with prior observations that heat treatment alone does not induce homotypic adhesion in lymphocytes in vitro [31]. To minimize the known nonspecific effects of thermal stress on hemodynamic parameters such as vasodilatation and increased blood flow [25, 26, 32], which could have an impact on lymphocyte-endothelial interactions during the observation period, heated mice were allowed to revert to normothermal core temperature before adoptive transfer of fluorescent-labeled lymphocytes. Given that the velocity of noninteracting lymphocytes passing through the venular tree was similar between normothermic and hyperthermia-pretreated groups, improved lymphocyteendothelial interactions were likely due to molecular changes in the endothelium and not from nonspecific hemodynamic thermal effects [25]. Taken together, evidence that a greater number of lymphocytes can recirculate through lymphoid organs over a given period of time with hyperthermia suggests that increased temperatures associated with fever directly benefit the process of primary immune surveillance.

A large range of molecules involved in the multistep trafficking cascade was evaluated to determine the causative agents of improved adhesion in HEVs [25]. Intravascular staining demonstrated ICAM-1 and CCL21 as the only components found to be substantially increased in terms of HEV lumenal expression. This increased expression could be quantified by image analysis of immunofluorescence, which indicated a $\sim 2$ - and $\sim 1$.5-fold increase over baseline immunofluorescence staining for ICAM-1 (Fig. 2) and CCL21, respectively, on near-cuboidal HEVs of PLNs and PPs [25]. In contrast, intravascular staining for other endothelial molecules such as ICAM-2 did not increase with heat arguing against a non-specific hemody-

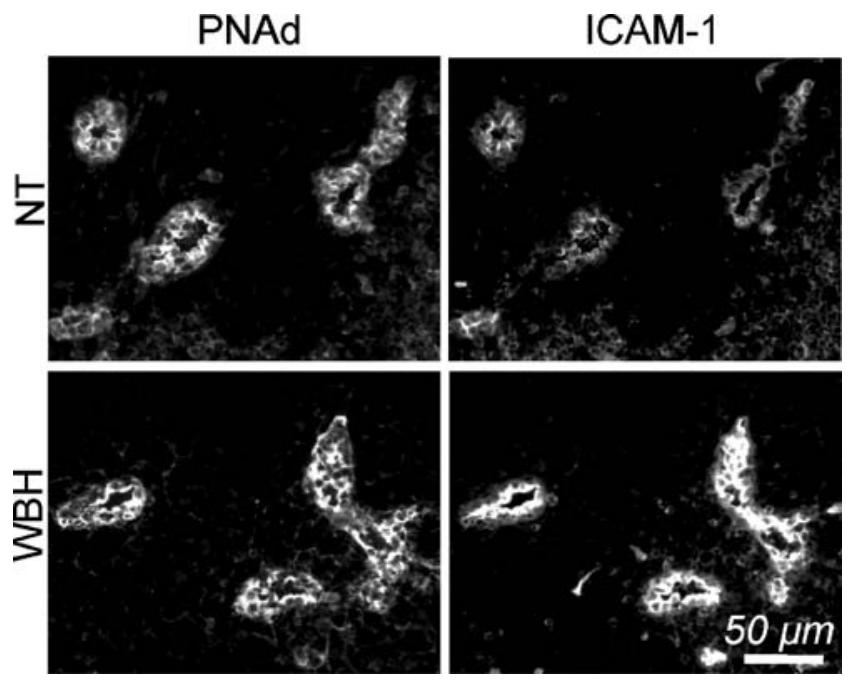

Fig. 2 Fever-range hyperthermia enhances the intravascular expression of ICAM-1 on PNAd ${ }^{+}$HEVs in peripheral lymph nodes. Intravascular ICAM-1 was detected by intravenous injection of ICAM-1-specific primary antibody followed by immunofluorescence staining. Peripheral lymph node cryosections were counterstained with MECA-79 antibody to demark the position of PNAd ${ }^{+}$near-cuboidal HEVs. The intravascular density of ICAM-1 was substantially upregulated in HEVs of mice receiving whole-body hyperthermia $(W B H)$ compared to normothermal $(N T)$ controls. PNAd expression was not affected by exposure to $\mathrm{WBH}$

namic effect on antibody delivery during staining procedures. Consistent with observations in homing assays and intravital microscopy studies, increased ICAM-1 and CCL21 staining was noted only in HEVs during WBH and not in normal squamous vascular endothelium of extralymphoid organs [25]. Upregulated CCL21 expression during $\mathrm{WBH}$ is functionally relevant to the improved trafficking of lymphocytes, as homing was abrogated by treating lymphocytes with pertussis toxin, a general inhibitor of chemokine receptor function [25]. Desensitization of CCR7 by ex vivo exposure of lymphocytes to high concentrations of CCL21 before adoptive transfer as well as in vivo antibody blockade of CCL21 similarly abolished the improved homing associated with WBH. Heat-inducible ICAM-1 was also demonstrated to be crucial for the observed increases in lymphocyte homing to lymph nodes [25] through the use of either ICAM-1 knockout mice or blocking antibodies to ICAM-1 in vivo.

Fever-range hyperthermia can be viewed as influencing lymphocytes early in the adhesion cascade (tethering/ rolling) as well as the endothelium in later steps (chemokine activation, firm adhesion, and transendothelial migration; Fig. 1). These mechanisms substantially increase the probability of antigen-specific $\mathrm{T}$ cells encountering the appropriate antigen-presenting cell in lymphoid organs. In a system where approximately one in four lymphocytes extravasate after entering HEVs under steady-state con- 
ditions [14], the approximately twofold increase observed with fever-range thermal stress represents a profound enhancement of primary immune surveillance. An interesting question that remains to be addressed is whether feverrange temperatures also increase the rate of transit of lymphocytes out of the lymph nodes via the efferent lymphatics [14].

\section{Elucidation of an IL-6 trans-signaling mechanism}

The mediator of thermal effects upon lymphocytes and HEVs was revealed in a series of complementary experiments. In vitro studies involving thermally treated lymphocytes implied that the hypothalamic responses associated with fever were not necessary for the changes seen with hyperthermia $[26,29,30,33]$. While thermal effects on lymphocyte adhesion were shown to be mediated by soluble factors in conditioned medium, no increase in concentrations of fever-associated cytokines such as interleukin (IL) $1 \alpha$, IL-1 $\beta$, IL-6, IL-8, or tumor necrosis factor (TNF) were detectable. Additionally, IL-2, IL-3, IL-4, IL10, IL-11, IL-12, IL-13, IL-15, interferon (IFN) $\gamma$, leukemia inhibitory factor, granulocyte monocyte colony-stimulating factor, transforming growth factor 1, and Flt3 were undetectable in this culture medium. These data suggested that thermal stress likely improves the bioactivity of soluble factors locally and not their overall concentrations. To determine the relevant cytokines, antibody blockade experiments against IL- 8 , IFN $\alpha$, IFN $\gamma$, TNF, IL- $1 \beta$, and IL-6 were performed. Only blockade of IL-6 was capable of neutralizing the effects of thermal stress on adhesion in lymphocytes treated either in vitro or in vivo [33].

IL-6 signaling can be triggered by two separate pathways [34]. In classical signaling, IL-6 influences cellular responses by binding to a specific IL- 6 cell surface receptor subunit (IL-6R $\alpha$ ). In turn, the IL-6R $\alpha /$ IL-6 complex initiates homodimerization of gp130, an associated molecule of the IL- 6 cell surface receptor. The gp130 complex is then capable of transducing the original IL-6 signal intracellularly [35]. An alternate signaling pathway for IL-6 utilizes a soluble form of the IL-6 receptor (sIL-6R $\alpha$ ). When bound to IL-6, the sIL-6R $\alpha$ can act to dimerize membrane-anchored gp130 leading to signal transduction. It is interesting to note that cells that do not express the membrane form of the IL-6R $\alpha$ but express gp130 are rendered responsive to the IL-6/sIL-6R $\alpha$ complex. This mode of action has been termed IL- 6 trans-signaling [35]. Accordingly, soluble forms of gp130 are capable of competitively inhibiting IL- 6 trans-signaling by binding to sIL-6R $\alpha /$ IL-6 complexes, thereby eliminating their interaction with membrane-bound gp130 [35].
In the models of hyperthermia-treated lymphocytes, the addition of antibodies against IL-6, the IL-6 receptor (which can recognize membrane or soluble forms), or gp130 similarly inhibited the increased adhesion associated with heat [33]. Moreover, blockade of IL-6 trans-signaling by the receptor antagonist, recombinant soluble gp130, prevented augmented heat-induced adhesion both in vitro and in vivo [33]. The role of IL-6 trans-signaling as the mediator of hyperthermia-induced lymphocyte changes was supported by the ability of recombinant soluble gp130 to inhibit the proadhesive effects of exogenously added IL-6 to lymphocyte cultures. Additionally, the level of membrane bound IL-6R $\alpha$ expression on treated lymphocytes was not detectable by flow cytometry [33]. Collectively, these lines of investigation support an IL-6 trans-signaling mechanism as being the prime mediator of enhanced lymphocyte adhesion during thermal stress.

Remarkably, a nonredundant requirement for IL-6 transsignaling was also demonstrated for the thermal induction of ICAM-1 on HEVs. By utilizing IL-6 knockout mice or administering IL-6 blocking antibody before WBH treatment of wild-type mice, the typical upregulation of ICAM1 expression on HEVs was eliminated [25]. To define the IL-6 pathway involved, recombinant soluble gp130 was injected into mice before WBH to selectively block IL-6 trans-signaling but not the classical pathway [25]. This treatment prevented ICAM-1 upregulation during WBH suggesting that IL-6 trans-signaling was the common mediator of thermal effects seen on both HEVs and lymphocytes. The increased expression of CCL21 seen with WBH, however, was not associated with an IL-6dependent pathway and remains to be elucidated [25].

\section{Clinical implications of thermal therapy dynamics}

The ability of fever-range WBH to selectively alter the adhesive properties of lymphocytes and endothelium has several potential clinical implications. The thermal component of fever appears beneficial to primary immune surveillance and may be hampered by the use of antipyretics. Subsequently, the judicious use of antipyretics is increasingly advocated [36]. Furthermore, the circulation of lymphocytes through lymph nodes is not only important during infection but may also be necessary for immune responses to cancer [37, 38]. Improved lymphocyte recirculation across HEVs by WBH treatment of cancer patients may allow for a more potent antitumor immune response based upon recent recognition that lymphoid organs are key sites for promoting antitumor immunity [39].

Another critical site of lymphocyte entry for elaboration of antitumor immunity is within the tumor microenvironment itself $[40,41]$. Tumor microvasculature in patient and 
murine models is associated with low basal levels of ICAM-1 expression, which may explain poor $\mathrm{T}$ cell infiltration in tumor tissues [32, 40, 42]. Recent studies have identified endogenous IL-6 and the soluble IL-6 receptor in local tumor microenvironments that were responsible for promoting tumor growth [43]. Paradoxically, IL-6 trans-signaling present in the tumor microenvironment may also be an attractive therapeutic target during systemic thermal therapy to inhibit tumor growth through immune-mediated mechanisms. It remains to be determined if hyperthermia can exploit IL-6 and the soluble IL-6 receptor in the tumor microenvironment to improve vascular adhesion similar to that observed in HEVs. In addition to changes in tumor blood flow and oxygenation associated with hyperthermia [44], improved vascular adhesion may theoretically enhance $\mathrm{T}$ lymphocyte infiltration into tumor sites, as has been reported for natural killer lymphocytes [22]. T lymphocyte infiltration is critical to antitumor responses and improved patient survival [45-47]. Although local hyperthermia in combination with other local intratumoral therapies such as vaccination, ablation, or radiation have potential efficacy by improving lymphocyte infiltration [48-50], the role of WBH alone as a modifier of the tumor microenvironment has not been established in the context of improving vascular adhesion. The limited toxicity of WBH in humans has already been borne out in initial clinical trials [27]. The ability to actively increase the delivery of tumor-reactive lymphocytes to the tumor microenvironment in immunotherapy protocols represents a current area of active investigation.

A working knowledge of the molecular mechanisms associated with the dynamic changes seen in lymphocyte trafficking during WBH may also benefit the current understanding of several inflammatory disease models. For example, lymphocyte recruitment to areas of inflammation has been linked to an IL-6 trans-signaling mechanism in autoimmune states such as rheumatoid arthritis and inflammatory bowel disease [34]. It is believed that IL-6 trans-signaling also triggers the transition from innate immunity to acquired immunity by selectively regulating chemokine and adhesion molecule-dependent recruitment of leukocyte subsets to inflamed tissues [51]. Thus, perturbations of this system may be responsible for the maintenance of a chronic inflammatory disease state [51]. Accordingly, directed and specific therapies such as IL-6 blocking antibodies and exogenous soluble gp130 are being evaluated for clinical use in chronic inflammatory disorders $[52,53]$.

Acknowledgments We thank Heinz Baumann and Elizabeth Repasky for long-standing contributions to this work and Michelle Appenheimer, Daniel Fisher, Jason Muhitch, Trupti Vardam, and Lei Zhou for critical review of the manuscript.

\section{References}

1. Paul WE (1999) Fundamental immunology. Lippincott-Raven, Philadelphia

2. Kluger MJ (1991) Fever: role of pyrogens and cryogens. Physiol Rev 71:93-127

3. Mackowiak PA (1994) Fever: blessing or curse? A unifying hypothesis. Ann Intern Med 120:1037-1040

4. Mackowiak PA (2000) Physiological rationale for suppression of fever. Clin Infect Dis 31(Suppl 5):S185-189

5. Covert JB, Reynolds WW (1977) Survival value of fever in fish. Nature 267:43-45

6. Bernheim HA, Kluger MJ (1976) Fever: effect of drug-induced antipyresis on survival. Science (New York, NY) 193:237-239

7. Hasday JD, Fairchild KD, Shanholtz C (2000) The role of fever in the infected host. Microbes Infect 2:1891-1904

8. Kluger MJ, Kozak W, Conn CA et al (1996) The adaptive value of fever. Infect Dis Clin North Am 10:1-20

9. Manthous CA, Hall JB, Olson D et al (1995) Effect of cooling on oxygen consumption in febrile critically ill patients. Am J Respir Crit Care Med 151:10-14

10. Schumacker PT, Rowland J, Saltz S et al (1987) Effects of hyperthermia and hypothermia on oxygen extraction by tissues during hypovolemia. J Appl Physiol 63:1246-1252

11. Schumacker PT, Rowland J, Saltz S et al (2001) Glossary of terms for thermal physiology Third Edition revised by The Commission for Thermal Physiology of the International Union of Physiological Sciences (IUPS Thermal Commission). Jpn J Physiol 51:245-280

12. Pritchard MT, Ostberg JR, Evans SS et al (2004) Protocols for simulating the thermal component of fever: preclinical and clinical experience. Methods (San Diego, Calif) 32:54-62

13. Kupper TS, Fuhlbrigge RC (2004) Immune surveillance in the skin: mechanisms and clinical consequences. Nat Rev 4:211-222

14. von Andrian UH, Mempel TR (2003) Homing and cellular traffic in lymph nodes. Nat Rev 3:867-878

15. Butcher EC, Picker LJ (1996) Lymphocyte homing and homeostasis. Science (New York, NY) 272:60-66

16. Rosen SD (2004) Ligands for L-selectin: homing, inflammation, and beyond. Ann Rev Immunol 22:129-156

17. Ebert LM, Schaerli P, Moser B (2005) Chemokine-mediated control of $\mathrm{T}$ cell traffic in lymphoid and peripheral tissues. Mol Immunol 42:799-809

18. Gunn MD, Tangemann K, Tam C et al (1998) A chemokine expressed in lymphoid high endothelial venules promotes the adhesion and chemotaxis of naive T lymphocytes. Proc Natl Acad Sci USA 95:258-263

19. Stein JV, Rot A, Luo Y et al (2000) The CC chemokine thymusderived chemotactic agent 4 (TCA-4, secondary lymphoid tissue chemokine, 6Ckine, exodus-2) triggers lymphocyte functionassociated antigen 1-mediated arrest of rolling $\mathrm{T}$ lymphocytes in peripheral lymph node high endothelial venules. J Exp Med 191:61-76

20. Carman CV, Springer TA (2004) A transmigratory cup in leukocyte diapedesis both through individual vascular endothelial cells and between them. J Cell Biol 167:377-388

21. Ostberg JR, Gellin C, Patel R et al (2001) Regulatory potential of fever-range whole body hyperthermia on Langerhans cells and lymphocytes in an antigen-dependent cellular immune response. J Immunol 167:2666-2670

22. Burd R, Dziedzic TS, Xu Y et al (1998) Tumor cell apoptosis, lymphocyte recruitment and tumor vascular changes are induced by low temperature, long duration (fever-like) whole body hyperthermia. J Cell Physiol 177:137-147

23. Roberts NJ Jr (1991) Impact of temperature elevation on immunologic defenses. Rev Infect Dis 13:462-472 
24. Ostberg JR, Repasky EA (2006) Emerging evidence indicates that physiologically relevant thermal stress regulates dendritic cell function. Cancer Immunol Immunother 55:292-298

25. Chen Q, Fisher DT, Clancy KA et al (2006) Fever-range thermal stress promotes lymphocyte trafficking across high endothelial venules via an interleukin 6 trans-signaling mechanism. Nat Immunol 7:1299-1308

26. Evans SS, Wang WC, Bain MD et al (2001) Fever-range hyperthermia dynamically regulates lymphocyte delivery to high endothelial venules. Blood 97:2727-2733

27. Kraybill WG, Olenki T, Evans SS et al (2002) A phase I study of fever-range whole body hyperthermia (FR-WBH) in patients with advanced solid tumours: correlation with mouse models. Int $\mathrm{J}$ Hypertherm 18:253-266

28. Stamper HB Jr, Woodruff JJ (1976) Lymphocyte homing into lymph nodes: in vitro demonstration of the selective affinity of recirculating lymphocytes for high-endothelial venules. J Exp Med 144:828-833

29. Wang WC, Goldman LM, Schleider DM et al (1998) Fever-range hyperthermia enhances L-selectin-dependent adhesion of lymphocytes to vascular endothelium. J Immunol 160:961-969

30. Evans SS, Schleider DM, Bowman LA et al (1999) Dynamic association of L-selectin with the lymphocyte cytoskeletal matrix. J Immunol 162:3615-3624

31. Evans SS, Bain MD, Wang WC (2000) Fever-range hyperthermia stimulates alpha4beta7 integrin-dependent lymphocyte-endothelial adhesion. Int J Hypertherm 16:45-59

32. Chen Q, Fisher DT, Kucinska SA et al (2006) Dynamic control of lymphocyte trafficking by fever-range thermal stress. Cancer Immunol Immunother 55:299-311

33. Chen Q, Wang WC, Bruce R et al (2004) Central role of IL-6 receptor signal-transducing chain gp130 in activation of L-selectin adhesion by fever-range thermal stress. Immunity 20:59-70

34. Jones SA, Horiuchi S, Topley N et al (2001) The soluble interleukin 6 receptor: mechanisms of production and implications in disease. FASEB J 15:43-58

35. Jostock T, Mullberg J, Ozbek S et al (2001) Soluble gp130 is the natural inhibitor of soluble interleukin-6 receptor transsignaling responses. Eur J Biochem 268:160-167

36. Plaisance KI, Mackowiak PA (2000) Antipyretic therapy: physiologic rationale, diagnostic implications, and clinical consequences. Arch Intern Med 160:449-456

37. Bai XF, Gao JX, Liu J et al (2001) On the site and mode of antigen presentation for the initiation of clonal expansion of CD8 T cells specific for a natural tumor antigen. Cancer Res 61:6860-6867

38. van Mierlo GJ, Boonman ZF, Dumortier HM et al (2004) Activation of dendritic cells that cross-present tumor-derived antigen licenses CD8+ CTL to cause tumor eradication. J Immunol 173:6753-6759

39. Gattinoni L, Klebanoff CA, Palmer DC et al (2005) Acquisition of full effector function in vitro paradoxically impairs the in vivo antitumor efficacy of adoptively transferred CD8+ T cells. J Clinical invest 115:1616-1626

40. Ganss R, Arnold B, Hammerling GJ (2004) Mini-review: overcoming tumor-intrinsic resistance to immune effector function. Eur J Immunol 34:2635-2641

41. Skitzki J, Craig RA, Okuyama R et al (2004) Donor cell cycling, trafficking, and accumulation during adoptive immunotherapy for murine lung metastases. Cancer Res 64:2183-2191

42. Chen Q, Wang WC, Evans SS (2003) Tumor microvasculature as a barrier to antitumor immunity. Cancer Immunol Immunother 52:670-679

43. Rose-John S, Scheller J, Elson G et al (2006) Interleukin-6 biology is coordinated by membrane-bound and soluble receptors: role in inflammation and cancer. J Leukoc Biol 80:227-236

44. Song CW, Park HJ, Lee CK et al (2005) Implications of increased tumor blood flow and oxygenation caused by mild temperature hyperthermia in tumor treatment. Int $\mathrm{J}$ Hypertherm 21:761-767

45. thor Straten P, Becker JC, Guldberg P et al (1999) In situ T cells in melanoma. Cancer Immunol Immunother 48:386-395

46. Hiraoka K, Miyamoto M, Cho Y et al (2006) Concurrent infiltration by $\mathrm{CD} 8+\mathrm{T}$ cells and $\mathrm{CD} 4+\mathrm{T}$ cells is a favourable prognostic factor in non-small-cell lung carcinoma. Br J Cancer 94:275-280

47. Galon J, Costes A, Sanchez-Cabo F et al (2006) Type, density, and location of immune cells within human colorectal tumors predict clinical outcome. Science (New York, NY) 313:1960-1964

48. Guo J, Zhu J, Sheng X et al (2007) Intratumoral injection of dendritic cells in combination with local hyperthermia induces systemic antitumor effect in patients with advanced melanoma. Int J Cancer 120:2418-2425

49. Morita M, Kuwano H, Araki K et al (2001) Prognostic significance of lymphocyte infiltration following preoperative chemoradiotherapy and hyperthermia for esophageal cancer. Int $\mathrm{J}$ Radiat Oncol Biol Phys 49:1259-1266

50. Nakayama J, Kokuba H, Kobayashi J et al (1997) Experimental approaches for the treatment of murine B16 melanomas of various sizes. I: Local injection of ethanol with a combination of interleukin-2 or microwaval hyperthermia for B16 melanomas with a size of less than $7 \mathrm{~mm}$ in diameter. J Dermatol Sci 15: $75-81$

51. Jones SA (2005) Directing transition from innate to acquired immunity: defining a role for IL-6. J Immunol 175:34633468

52. Paul-Pletzer K (2006) Tocilizumab: blockade of interleukin-6 signaling pathway as a therapeutic strategy for inflammatory disorders. Drugs Today (Barc) 42:559-576

53. Nowell MA, Richards PJ, Horiuchi S et al (2003) Soluble IL-6 receptor governs IL-6 activity in experimental arthritis: blockade of arthritis severity by soluble glycoprotein 130. J Immunol 171:3202-3209 